\title{
Confidence is epistemic probability for empirical science
}

\author{
Tore Schweder \\ Department of Economics \\ University of Oslo \\ Norway
}

August 18, 2017

\begin{abstract}
In its orthodoxy standard frequentist statistics deals only with aleatory probability, suppressing the intuitive epistemic probability representing inferential uncertainty. Confidence distributions, which are posterior distributions not based on any Bayesian priors, are discussed in nontechnical terms, with emphasis on the confidence curve. The correspondence between confidence curves and likelihoods allows independent confidence curves and confidence intervals to be integrated. Confidence and (serious) $p$-values are interpreted as epistemic probabilities, which do not fully follow ordinary probability calculus. Dimension reduction and other operations might be done on the likelihood related to the confidence curve. Confidence distributions and objective Bayes have much in common.
\end{abstract}

Keywords Confidence distribution; Confidence curve; $P$-value; Neyman-Pearson; Likelihood; Objective Bayes

\section{Introduction}

In this short essay I suggest that the confidence of confidence intervals and confidence distributions is a concept of epistemic probability. A $p$-value for significance testing being a confidence obtained from a confidence distribution is also understood as an epistemic probability. These epistemic probabilities are interpersonal since they only depend on the model and the data. Whenever the model and the data are accepted, confidence is the appropriate probability representing inferential uncertainty - in the context of statistical inference in empirical science.

The word 'probability' has a long and complex history. Before the Renaissance Probability, from the Latin 'probabilitas', was used as an ordinal measure of authority: "worthy of approbation" (Hacking 1975, p. 18). The German Wahrscheinlichkeit is "true-seemingness', the same as the Scandinavian Sannsynlighet. Probability was originally a purely epistemic concept. It was mostly qualitative, ordering statements with respect to degree of belief, and their epistemic probability or their weight was based on knowledge and/or authority. 
Hacking $(1975$, p. 73$)$ dates the modern concept of probability to the year 1662 when La logique ou l'art de penser (Arnauld, and Nicole 1662) was published from the abbey Port Royal to which Blaise Pascal was associated. From that year attempts were made to quantify probability by mathematical arguments, particularly by counting the favorable outcomes for an event in an equi-probable model. Probability thus got its Janus face. Epistemic probabilities of parameters or statements characterizing the uncertainty surrounding our knowledge, obtained from mathematical argument and/or empirical observations, faced one way. Aleatory probability representing chances of events in real experiments or processes in society or nature, thought of as long-term frequencies in repeated experiments, faced the other way.

Epistemic probability can be subjective, intersubjective or logical. The subjective Bayesian uses Bayes' lemma to update her personal probability distributions in view of the data and the model. When IPCC states "It is extremely likely that more than half of the observed increase in global surface temperature from 1951 to 2010 was caused by the anthropogenic increase in greenhouse gas concentrations and other anthropogenic forcings together" (IPCC 2013, Summary for Policymakers, p. 17), and codifies 'extremely likely' to be more than $95 \%$ probable, they employ an intersubjective concept of epistemic probability. Fisher's fiducial probability distributions, and also their generalizations (Hannig et al. 2016) as well as the ingenious inferential models of Martin and Liu (2015), which often are confidence distributions, represent logical epistemic probabilities. Kalbfleisch and Sprott (1967) say that "the fiducial distribution of $\theta$ is not a distribution of belief, but a summary of the information about $\theta$ provided by the observations which one is free to believe or disbelieve". Fisher would probably have agreed to the first part of the statement, but he would have added that the rational mind would then have the fiducial distribution as knowledge, and in that sense regard it as his or her distribution of belief obtained by pure logic.

The basically dual nature of probability has not been easy to handle. The field of statistics has been swinging between epistemic and aleatory probability. From the time of Bayes and Laplace, probability has mostly been understood as epistemic. The Fisherian revolution around 1920 (Hald 1993, p. 1), followed by works of Neyman, Pearson, Wald and others, swung probability to be purely aleatory. In the last half-century the Bayesian tradition has been revitalized, and statistical inference is now split between frequentists and Bayesians, i.e. roughly between those who understands probability as aleatory and those who see it as epistemic. The division of the field in these two camps is unfortunate.

The estimation of model parameters is an essential part of empirical science. There are also other statistical aspects of empirical science, but we shall concentrate on estimation, including the assessment of the uncertainties surrounding point estimates. The Bayesian posterior distribution is an eminent representation of what has been learned and how uncertain this knowledge is, provided the prior distribution used to obtain the posterior really represents the a priori information available. When this provision is in doubt the Bayesian posterior will also be in doubt. This difficulty had bothered authors in the 19th Century, see e.g. Boole (1854 p.370). It led Fisher (1930) to suggest his fiducial distribution for a scalar parameter. The great virtue of the fiducial distribution is that it often represents an epistemic distribution for a parameter based on the data and the model, without any prior distribution.

Neyman (1934) was enthusiastic about Fisher's fiducial distribution, not the least because it is useful to obtain confidence intervals. Not all fiducial distributions lead to 
confidence regions, however, see Example 3 below. A distribution for the parameter, based only on the data and the model, and which leads to confidence regions is called confidence distribution. Confidence distributions, and particularly their confidence curves, are compact representations of families of confidence regions for (vector) parameters. Confidence distributions are thus posterior distributions obtained without any prior. Bayesian distributions are often approximate confidence distributions, and Fraser (2011) asks whether Bayes posteriors are just quick and dirty confidence distributions. If we could agree that confidence is an important property of epistemic distributions in cases where legitimate priors are unavailable, the unfortunate split between the Bayesian and the frequentist camp might be lessened.

But is this split necessary? Efron (1998) hopes not, and thinks that Fisher's idea of fiducial distributions might bridge the gap. He says, "My actual guess is that the old Fisher will have a very good 21st century. The world of applied statistics seems to need an effective compromise between Bayesian and frequentist ideas, and right now there is no substitute in sight for the Fisherian synthesis."

Fiducial distributions were largely neglected by statisticians in the last half-century because they were oversold by Fisher, but presently receives considerable attention, see e.g. Cisewski and Hannig (2012) and Hannig et al. (2016). Fiducial distributions are exact or approximate confidence distributions. Emphasizing the epistemic probability interpretation of the confidence distribution and the confidence curve, these concepts might thus be the basic concepts in the Fisherian synthesis that Efron hopes for.

After a brief introduction to confidence distributions and confidence curves, I will hook on to the current debate (Trafimow and Marks, 2016; Wasserstein and Lazar, 20016) over $p$-values. When used properly, I see them as legitimate epistemic probabilities. There is however a lot of misuse. The next section presents some Neyman-Pearson optimality theory for confidence distributions. A method for combining independent confidence distributions and confidence intervals by way of their related pseudo-likelihoods is also mentioned. Before concluding I discuss briefly the relationship between Bayesianism and inference with confidence distributions, and note that the relation is particularly close when Objective Bayes is used. The aim of the essay is to encourage the use of statistical inference by way of confidence distributions, and to think of confidence as epistemic probability.

\section{Confidence distributions}

The term 'confidence distribution' was first used by Cox (1958). If for each $\alpha \in(0,1)$, $(-\infty, \theta(\alpha, X)]$ is a half-open confidence interval of degree $\alpha$ for the parameter $\theta$, based on the data $X$ generated by the accepted model, the endpoints $\theta(\alpha, X)$ are the quantiles of the confidence distribution. Thus, $C(t, x)=\theta^{-1}(t, x)$ as a function of $t$ for given $x$ is the cumulative confidence distribution function for observed data $x$, and $C(t, X)$ is its stochastic counterpart, with standard uniform null distribution,

$$
C(\theta, X) \sim \mathrm{U}(0,1)
$$

when $X$ is continuously distributed according to $\theta$. Cox (2013) sees confidence distributions as "simple and interpretable summaries of what can reasonably be learned from the data (and an assumed model)". 
Example 1 (Confidence distribution for a a location parameter) Let $X$ have density $f(x-\theta)$ where $f$ with $c d f F$ is known. Then $1-F(X-\theta)$ is uniformly distributed, and $1-F(x-\theta)$ is a cumulative distribution function in the location parameter for each $x$. Thus $C(\theta, x)=1-F(x-\theta)$ is a realized cumulative confidence distribution for $\theta$, with density $c(\theta, x)=f(x-\theta)$. This is the unique (realized) confidence distribution in this simple model. Its $\alpha$ quantile for given $x$ is $C^{-1}(\alpha)=x-F^{-1}(1-\alpha)$, and $\left(C^{-1}(\alpha / 2), C^{-1}(1-\alpha / 2)\right)$ is the tail-symmetric confidence interval of degree $1-\alpha$. The Bayesian obtains the same posterior distribution when using a flat improper prior.

Confidence distributions are reviewed by Xie and Singh (2013) and more extensively by Schweder and Hjort (2016).

Birnbaum (1961) introduced the two-sided 'confidence curve' $c c(\theta, X)$ with the defining property of having level sets $\{\theta: \operatorname{cc}(\theta ; X) \leq \alpha\}$ as confidence intervals of degree $\alpha \in(0,1)$. By presenting the confidence curve, and thus confidence intervals of all levels, the reader is given a complete picture of the inferential uncertainty, see Example 4 below.

The concept of confidence curve extends to vector parameters where the level sets are confidence regions. The defining property for confidence curves is that

$$
c c(\theta, X) \sim \mathrm{U}(0,1)
$$

when $X$ is distributed according to $\theta$. By this property the level sets have the correct coverage probability:

$$
P_{\theta}(\{t: c c(t, X) \leq \alpha\} \ni \theta)=P_{\theta}(c c(\theta, X) \leq \alpha)=\alpha .
$$

In case there is a nuisance parameter (vector) $\chi$ in addition to the the parameter of interest $\theta$, an exact confidence curve for the latter has the property mentioned above, $c c(\theta, X) \sim \mathrm{U}(0,1)$ for all $(\theta, \chi)$.

Example 2 (Normal model) The archetypal confidence curve is the normal confidence curve based on an estimate $\hat{\mu}$ assumed normally distributed about $\mu$ with known standard error $\sigma$. The curve $c c(\mu, \hat{\mu})=|1-2 \Phi((\mu-\hat{\mu}) / \sigma)|$ is seen to yield as level sets at degree of confidence $\alpha=1-\epsilon$ the familiar normal confidence intervals $\left(\hat{\mu}+\sigma z_{\epsilon / 2}, \hat{\mu}+\sigma z_{1-\epsilon / 2}\right)$. Here $z_{p}=\Phi^{-1}(p)$. This confidence curve is a symmetric funnel plot pointing down at the point estimate. It is tail-symmetric in the sense that its confidence intervals misses their target with the same epistemic probability to each side. The width of the confidence curve is proportional to $\sigma$. The tighter the confidence curve the more informative it is.

Note that a cumulative confidence distribution $C(\theta, X)$ is a one-sided confidence curve for the family of half-open confidence intervals. The canonical two-sided confidence curve for a confidence distribution $C$ is

$$
c c(\theta, X)=|1-2 C(\theta ; X)|,
$$

with tail-symmetric confidence intervals $(\theta(\epsilon / 2, X), \theta(1-\epsilon / 2, X))$ at level $\alpha=1-\epsilon$

By abusing notation slightly, I will often write $C$ for a realized confidence distribution of observed data $x$. The same for a confidence curve and a confidence density. Their stochastic counterparts are written out as $C(\theta, X)$ etc.

A confidence curve will not determine a unique cumulative confidence distribution, without further information. If, for example, it is known that the confidence curve has 
only one local minimum and is tail symmetric, the confidence density is $c(\theta)=\frac{d}{d \theta} C(\theta)=$ $\frac{1}{2}\left|\frac{d}{d \theta} c c(\theta)\right|$. But two-sided confidence curves need not be tail symmetric. Despite this lack of uniqueness I will let a confidence distribution be represented by one of its confidence curves, in dimension 1 preferably the canonical tail symmetric one. Actually, I will further abuse notation slightly by sometimes using the term 'confidence distribution' for 'confidence curve', even when there are no proper distribution behind the confidence curve. By letting confidence curve being the basic concept, more general distributional inference is possible than if a distribution, possibly improper, is required as the inferential result.

As argued above, level sets $\{\theta: c c(\theta, X) \leq \alpha\}$ are confidence regions for $\theta$ of degree $\alpha$. This was the reason for Neyman (1934) to be so enthusiastic about Fisher's fiducial distributions, although the confidence curve was only suggested by Birnbaum (1961). By emphasizing the confidence curve, I thus follow Neyman's interpretation of Fisher's fiducial distribution. I do however regard the confidence as epistemic probability $e x$ post, i.e. $C(\theta, x)$ is the cumulative epistemic probability distribution when the observed data are $x$. Ex ante, before the data are obtained the confidence distribution is an aleatory stochastic element $C(\theta, X)$, with the important property of having control over the coverage probability of any confidence region it yields. The Neyman-Pearson school of statistics teaches however that there is only one form of probability, namely the aleatory, and refuses to interprete the confidence level as an epistemic probability ex post.

The statement "the Newtonian gravitational constant $\gamma$ is between 6.6723 and 6.6754 (in appropriate units) with probability 0.95 " is in my view perfectly meaningful. It is based on the best available evidence (Milyukov and Fan, 2012) from where this 95\% confidence interval for $\gamma$ is obtained. The statement is however not acceptable to orthodox followers of the Neyman-Pearson school. They would argue that there is no (hypothetical) experiment behind this probability statement, and with only aleatory probability at hand the statement has no meaning. Hampel (2006) disagrees: "But as probably most of those of us know who tried to teach Neyman-Pearson statistics to critical, intelligent, unspoilt users of statistics, these scientists have a strong intuition that even "after the fact" there is, or should be, something with $95 \%$ probability; and they are very frustrated when they are told their intuition is entirely wrong." It is the exclusive use of aleatory probability that is the root of the error, he thinks, and I agree. The normal confidence distribution centered at 6.67384 , and with standard deviation 0.00080 is "what can reasonably be learned" (Cox, 2013) from the data reviewed by Milyukov and Fan (2012). (The normal error distribution, which I assume, is however not explicitly mentioned in the source.) The realized confidence distribution is clearly an epistemic probability distribution, and the epistemic probability is in this case of the logical type.

Confidence curves might be constructed in different ways. One approach is to transform the profile deviance to be uniformly distributed at the true value of $\theta$. For the observed data $x$ the profile $\log$-likelihood is $\ell_{\text {prof }}(\theta, x)=\max _{\chi} \log f_{(\theta, \chi)}(x)$, and the profile deviance regarded as a stochastic element $D_{\text {prof }}(\theta, X)=-2\left(\ell_{\operatorname{prof}}(\theta, X)-\max \ell_{\text {prof }}(\theta, X)\right)$ is approximately $\chi^{2}$-distributed with $p$ degrees of freedom by the Wilks Theorem (Schweder and Hjort p.39). With $K_{p}$ the cumulative distribution function of the $\chi^{2}$ distribution of $p$ degrees of freedom,

$$
c c(\theta, X)=K_{p}\left(D_{\text {prof }}(\theta, X)\right)
$$

is thus an approximate confidence curve. 
The confidence curve (1) may be named the maximum likelihood confidence curve. Not only does it point at the maximum likelihood estimator as its minimum but it also have level sets and thus confidence regions consisting of parameter values with likelihood above a certain value and excluding points of likelihood below that value. There are confidence curves that share these properties, having more complex level sets than single intervals, e.g. (2) below.

Epistemic probability in the form of confidence or fiducial probability does unfortunately not in general follow ordinary probability calculus, despite the old Fisher (1973, p. 59) claiming "There are in reality no grounds for any such distinction" between fiducial and ordinary aleatory probability. A distribution derived from a confidence distribution is actually not necessarily itself a confidence distribution, as demonstrated by the following simple example.

Example 3 (Confidence curve for $|\mu|$ in the normal model.) The cumulative confidence distribution for $\mu$ in Example 1 is $C(\mu)=\Phi((\mu-\hat{\mu}) / \sigma)$. The cumulative distribution for $\theta=|\mu|$ derived from this is thus $H(\theta)=\Phi((\theta-\hat{\mu}) / \sigma)-\Phi((-\theta-\hat{\mu}) / \sigma)$. This is however not a confidence distribution since $H(\theta, \hat{\mu})$ can be far from uniformly distributed on the unit interval. When, say, $\theta=1$ the distribution is actually supported on the interval $(0,0.7)$, and is not even uniform on this interval. From the distribution of $\hat{\theta}$, the confidence curve $c c(\theta)=|1-2(\Phi((\hat{\theta}-\theta) / \sigma)-\Phi((-\hat{\theta}-\theta) / \sigma))|$ is obtained. It is plotted in Figure 1 for $\hat{\theta}=1$, the maximum likelihood estimate. This realized confidence distribution has a point mass of 0.32 at $\theta=0$. Confidence intervals of level more than 0.36 will thus have the lower limit $\theta=0$ as their left endpoint. Note that the confidence curve is minimal slightly to the left of $\hat{\theta}$. It actually points at the median unbiased estimate $C^{-1}(1 / 2)$.

Some authors have worked toward a calculus for confidence distributions. Pitman $(1939,1957)$ suggested conditions for when confidence distributions can be obtained from another confidence distribution by ordinary probability calculus. Hacking (1965, p. 147) put forward some other principles. My view is that we still are far from having a mathematical theory of confidence calculus. Such a theory would be wonderful to have, but I am unfortunately pessimistic about the possibility of ever obtaining one. At present the best way forward is, in my view, to make use of our impressive theory of likelihood (see e.g. Brazzale et al. 2007), and to adapt it to confidence curves. My experience is actually also that it often is slightly simpler and more natural to phrase asymptotic theory in terms of confidence distributions than in terms of maximum likelihood estimators because solving the normal equation is avoided (Schweder and Hjort 2016, Chapter 7).

In Example 3 the confidence distribution has an atom at zero. This is not uncommon for parameters that have a restricted range. Traditionally Bayesians do not use priors with atoms, and will thus end up with posteriors without atoms. They will most often also end up with posteriors that are proper distributions. In the next example the confidence curve turn out to be more complex than a simple funnel, and is thus not representing a proper distribution. This distribution cannot be represented by a cumulative distribution function or a density. Despite this I call it a distribution since it distributes confidence over regions in the parameter space.

Example 4 (Confidence curve for a ratio of two normal means) For $\hat{a} \sim N\left(a, \sigma_{a}^{2}\right), \hat{b} \sim$ $N\left(b, \sigma_{b}^{2}\right)$ the profile deviance (assuming independence, and the variances to be known) for 


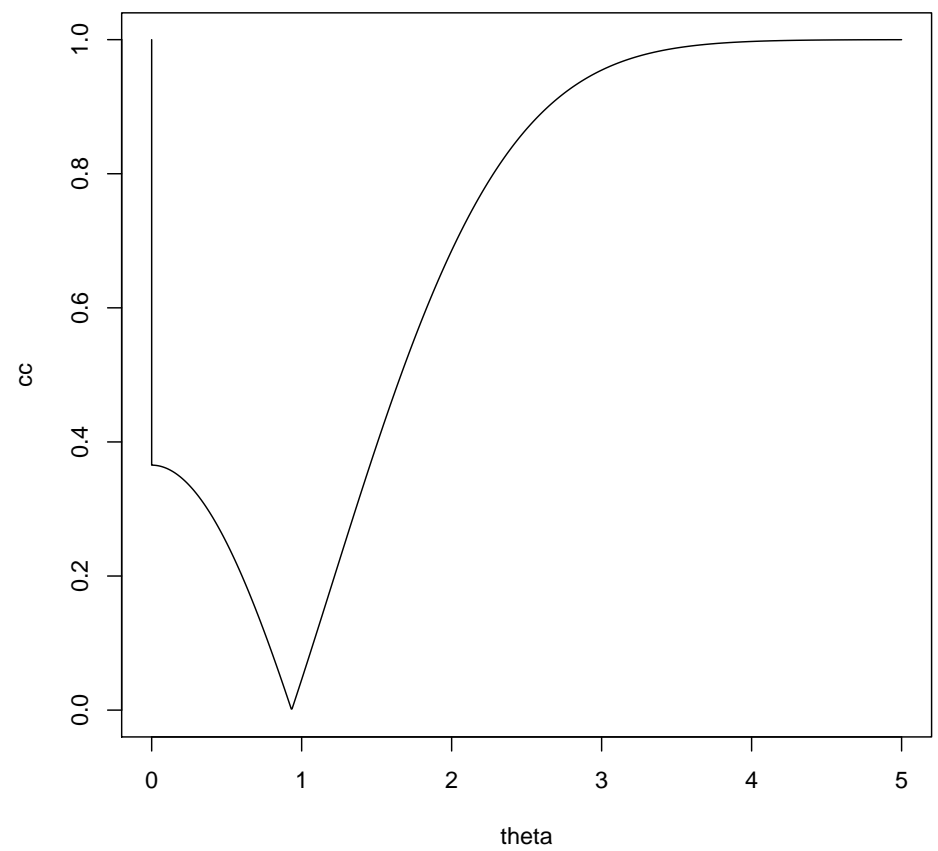

Figure 1: Confidence curve for $\theta=|\mu|$ based on a normal observation $\hat{\mu}=1$ of standard error $\sigma=1$. 
the ratio $\theta=a / b$ is

$$
D_{\text {prof }}=\frac{(\hat{a}-\theta \hat{b})^{2}}{\sigma_{a}^{2}+\theta^{2} \sigma_{b}^{2}} .
$$

This profile deviance is exactly $\chi_{1}^{2}$ at the true value of the ratio parameter, and

$$
c c(\theta)=K_{1}\left(D_{\text {prof }}(\theta)\right)
$$

is an exact confidence curve. The confidence curve obtained for $\theta$ when $\hat{a}=1.333, \hat{b}=$ 0.333, $\sigma_{1}=\sigma_{2}=1$ is shown in the left panel of Figure 2, while the right panel shows its contour tree which summarizes its structure and that of its confidence sets. The confidence curve has a maximum of 0.831 at $\theta \approx-1 / 4$. Above this level all confidence regions equal the whole real line. The data are thus too weak to say anything about $\theta$ with confidence higher than .831. Below this level, confidence regions are drawn by horizontal lines at levels $0.1, \ldots, 0.8$. At intermediate levels the confidence regions are seen to be unions of two disjoint intervals. The confidence curve tells us that the evidence in these data for $\theta$ is split between positive intervals and negative intervals at intermediate confidence. At confidence below 0.3 the evidence is contained in intervals around the maximum likelihood estimate of $\hat{\theta}=4.003$. As confidence tends to zero the confidence intervals shrink towards this single point.

\section{$3 \quad P$-values and the quiet scandal of statistics}

As confidence intervals are found from a confidence distribution, so are $p$-values for most testing problems. For a scalar parameter $\theta$ with confidence distribution $C$ the $p$-value for the null hypothesis $\theta \leq 0$ is the confidence of the interval $(-\infty, 0], C(0)$. As the level of a confidence interval should be interpreted as the epistemic probability of the true parameter value being inside the interval the $p$-value is the epistemic probability of the null hypothesis $\theta \leq 0$ being true.

Significance tests and confidence sets are closely related. For a completely specified $H_{0}: \theta=\theta_{0}$ against the alternative $\theta \neq \theta_{0}$, where the parameter might be a vector, it is usually not appropriate to view the $p$-value as the confidence of the null hypothesis. When based on a test-statistic $T$, and $p$-value $p_{\theta_{0}}=P\left(T \geq T_{o b s}, \theta_{0}\right)$, the set $S_{\alpha}=\left\{\theta_{0}: p_{\theta_{0}} \geq \alpha\right\}$ is actually a confidence set of level $1-\alpha$. A nested family of confidence sets is represented by a confidence curve. The confidence curve behind $\left\{\theta_{0}: p_{\theta_{0}} \geq \alpha\right\}, \alpha \in(0,1)$ has level sets indexed by the test-statistic $T$. The $p$-value of $H_{0}: \theta=\theta_{0}$ should thus be interpreted as the confidence of the statement "the null hypothesis is possibly true", while $1-p$ is the confidence of the hypothesis not being true. The $p$-value is is thus an epistemic probability. This accords with Martin and Liu (2014) who finds that a $p$-value can always be obtained as a plausibility in some inferential model.

The provision for the $p$-value being an epistemic probability is of course that it is legitimate and is not, say, selected as the "most significant result" among many, or is obtained from the selected model without accounting for the model selection process. As the confidence curve evaluated at the true parameter value ex ante is uniformly distributed, the $p$-value for $H_{0}: \theta=\theta_{0}$ is uniformly distributed under the null hypothesis. Model selection is known to often shift the distribution of the $p$-value to lower values when not accounted for. This is unfortunately not taken note of in much applied statistical work, 

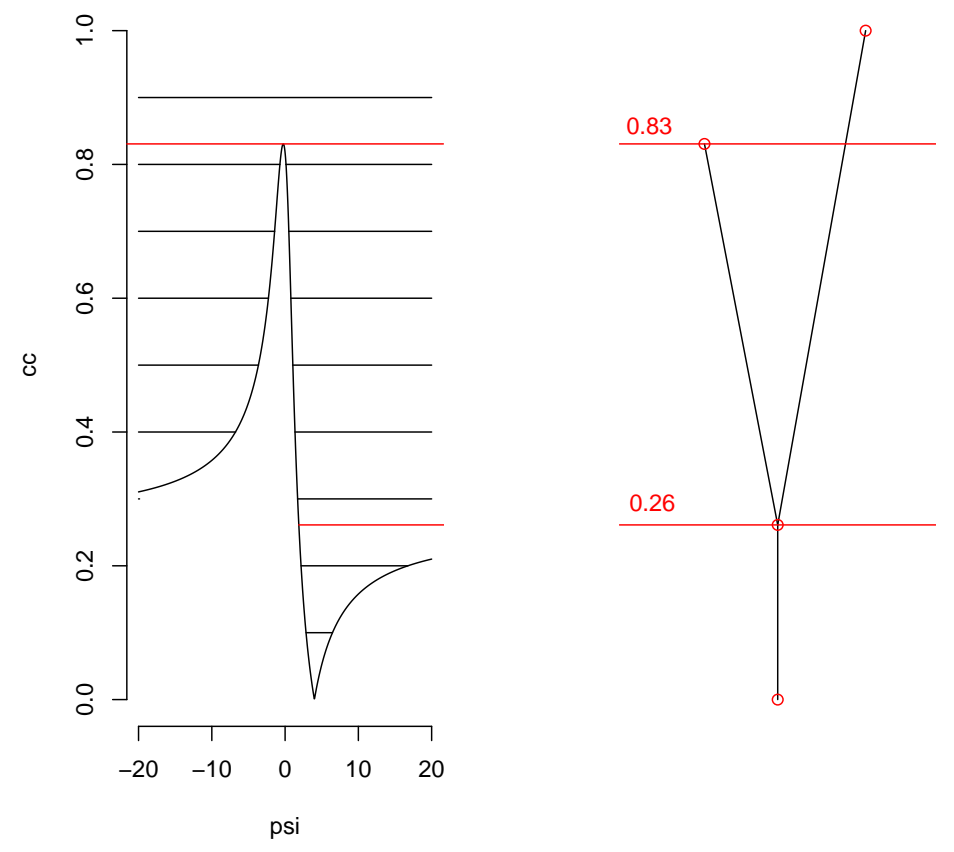

Figure 2: Left panel, confidence curve in Example 4 for $\psi=a / b$ based on independent normally distributed estimates $\hat{a}=1.333, \hat{b}=0.333$, both with known standard errors of one. Confidence regions at levels $0.1,0.2, \ldots, 0.9$. are shown with horizontal lines. Above 0.831 the whole real line are the level sets. Right panel, the contour tree of the confidence curve. 
even when carried out by experienced statisticians. This concerned Breiman (1992) and he called it "the quiet scandal of statistics". Other conscious or unconscious searches for "strong" $p$-values will also shift their null distributions to the left, possibly quite dramatically so. The widespread use of such illegitimate $p$-values is a not so quiet scandal of empirical science.

In 2015 the editors of Basic and Applied Social Psychology (BASP) announced that the journal would no longer publish papers containing $p$-values, because the values were too often used to support lower-quality research. The editors (Trafimow and Marks, 2016) state "The probability of the finding (or one more extreme) given the null hypothesis (p) is not the same as the probability of the null hypothesis given the finding, nor does $p$ provide a strong basis for drawing conclusions about the probability of the null hypothesis given the finding. Without a strong basis for drawing a conclusion about the probability of the null hypothesis given the finding, there is little justification for rejecting the null hypothesis simply because $\mathrm{p}<.05$. This rejection - the heart of the null hypothesis significance testing procedure - commits the inverse inference fallacy." They go on with "The proper-use claim: Some researchers argue that the many problems critics have associated with the use of $\mathrm{p}$ are due to misuse of $\mathrm{p}$. If it were not misused, these researchers claim, $p$ would be valuable. The problem with this argument is that no one who has made it has explained what that value is. What conclusions can validly be drawn from $\mathrm{p}$, other than the conclusion that $\mathrm{p}$ provides the probability of the finding (or one more extreme) given the null hypothesis, which is true by definition and hence trivial? We are not told. As we have explained in the foregoing bullet points, any use of $p$ to draw conclusions about hypotheses, replication, the role of chance, and so on, constitutes misuse. Of course $p$ is not misused if no conclusions are drawn from it. But if the only way to avoid misusing $p$ is to draw no conclusions from it, then wherein lies its value? We are not told. (...) The ban on $p$ values will continue."

Misuse aside, a properly obtained $p$-value of a completely specified hypothesis of scientific interest is our epistemic probability of the hypothesis possibly being true, given the statistical model and the observed data. If the confidence of the hypothesis being false, $1-p$, is sufficiently high there is good reason to suggest that the hypothesis should be rejected. This is in my view a valuable summary of the data regarding the hypothesis, and is what traditionally has been taught in basic courses in statistics, but without mentioning epistemic probability and with emphasize on $p$ rather than $1-p$. The editors of BASP do not understand $p$-values this way. They are not alone. My view of what serious $p$-values really are is actually also at odds with the official view of the American Statistical Association (ASA).

ASA (Wasserstein and Lazar 2016) states among other things: "P-values do not measure the probability that the studied hypothesis is true, or the probability that the data were produced by random chance alone. Researchers often wish to turn a p-value into a statement about the truth of a null hypothesis, or about the probability that random chance produced the observed data. The p-value is neither. It is a statement about data in relation to a specified hypothetical explanation, and is not a statement about the explanation itself." This is the first and only official statement on statistical methodology issued from ASA, and should not be taken lightly, but I disagree. Reid and Cox (2015) also note the widespread criticism against interpreting p-values and confidence as epistemic probabilities, "although the increase in general statistical literacy seems to have assuaged this concern somewhat." 
There is still wide disagreement and confusion over what $p$-values are. I think that researchers are right to view their $p$-value obtained by serious work as their epistemic probability, not necessarily of their hypothesis being true but of it possibly being true. In the frequentist tradition of Neyman-Pearson there is however no room for epistemic probability. This is probably a root of our problems with significance testing and $p$-values.

\section{Working with both aleatory and epistemic probabil- ity}

Despite its name, epistemic probability understood as confidence does not in general follow the ordinary rules of probability calculus. This unfortunate fact was mentioned above. The distribution of a derived parameter obtained from a confidence distribution might actually not be a confidence distribution, as shown in Example 3, where it also was noted that the implied point estimate $\tilde{\theta}$ is different from the maximum likelihood estimate. As we know, confidence intervals are invariant to monotone transformations of

the parameter. So also with confidence curves, and thus also their minimal point $\tilde{\theta}$. It is actually legitimate to transform confidence distributions monotonically.

Some other probability calculations can also be done on confidence distributions. When the confidence distribution is a proper distribution, like the above $C(\theta, x)=$ $\Phi(\theta-x), \theta_{C D}(x)=C^{-1}(U, x), U \sim \mathrm{U}(0,1)$ is what is called a confidence random variable (Schweder and Hjort 2016, p. 138). Its median $\tilde{\theta}=\operatorname{med}\left(\theta_{C D}(x)\right)=C^{-1}(1 / 2, x)$, is a median unbiased point estimate. We have actually that $\operatorname{med}\left[\operatorname{med}\left(\theta_{\mathrm{CD}}(\mathrm{X})\right)\right]=\theta$, where the outer median is with respect to the distribution of the data, which usually is aleatory, and the inner one is with respect to the epistemic confidence distribution.

The spread of a proper one-dimensional confidence distribution may be defined through a non-decreasing penalty function $\Gamma, \Gamma(0)=0$, providing the loss function $\operatorname{lo}(\theta, \mathrm{x})=$ $\left.\mathrm{E}\left\{\mid \Gamma\left(\theta_{\mathrm{CD}}(\mathrm{x})-\theta\right)\right) \mid\right\}$, where the expectation is over the confidence distribution for given data $x$, and the risk function $R(\theta)=\mathrm{E}\{\operatorname{lo}(\theta, \mathrm{X})\}$ which is the expected confidence spread with respect to the sampling distribution. The loss and the risk may be evaluated via the confidence curve $c c(\theta)=|1-2 C(\theta)|, \operatorname{lo}(\theta, \mathrm{x})=\int \Gamma\left(\theta^{\prime}-\theta\right) \operatorname{cc}\left(\mathrm{d} \theta^{\prime}\right)$. Again we mix aleatory and epistemic probability concepts.

Example 5 (Location model, continued) In the location model of Example 1, the risk is $\mathrm{R}(\theta)=2 \operatorname{varX}$ when penalty is quadratic, $\Gamma(t)=-\Gamma(-t)=t^{2}, t \geq 0$. To see this, note that in this location model $\theta_{C D}(X)=X-F^{-1}(1-U)$ where the uniform random variable $U$ is independent of $X$. Thus $E\left(\theta_{C D}(X)-\theta\right)^{2}=E(X-\theta)^{2}+E\left(F^{-1}(1-U)\right)^{2}=$ $2 \mathrm{varX}$. Half the risk is due to the variance in the realized confidence distribution and the other half is due to the sampling variation. This is a general phenomenon. The variation in the confidence distribution reflects the sampling variability.

\subsection{An optimality property}

The smaller the spread in a confidence distribution the more informative it is. The loss function measures the spread in a realized confidence distribution.

The Neyman-Pearson optimality result is usually phrased in terms of hypothesis testing. It can also be cast in terms of confidence intervals. It is thus no surprise that there 
are versions of Neyman-Pearson optimality for confidence distributions. Schweder and Hjort (2016, Chapter 5) prove several versions of Neyman-Pearson optimality, among them the following.

Theorem 6 (Neyman-Pearson for confidence distributions) A confidence distribution based on a sufficient statistic in which the likelihood ratio is everywhere increasing, is uniformly optimal in the sense that its loss function is less than or equal to that of any competitor with probability one - for all penality functions $\Gamma$ and for all $\theta$.

It is surprising that the optimality is with probability one, and not only that the risk is uniformly optimal.

As a corollary, the conditional confidence distribution for a one dimensional parameter $\theta$ given the ancillary statistic in an exponential family model with a $q$-dimensional nuisance parameter, is uniformly optimal (Schweder and Hjort 2016 Chapter 5).

Loss and risk should not be taken too literally. Guttman (1985) says "Science has no special loss function which is to be minimised. And scientific hypotheses are always open to be retested. Research in science should be cumulative. In the course of accumulation, previous hypotheses become modified by correction and extensions. The idea of accepting or rejecting a null hypothesis on the basis of a single experiment - which is the crux of the Neyman-Pearson theory - is antithetical to science." Confidence distributions are good for cumulative science. To get the most informative estimate out of an observed set of data, the best possible confidence distribution should be used. Confidence loss and risk for a particular penalty function might not be of much interest in itself, but uniform optimality should be of interest, although achieved in very simple models only.

\subsection{Combining confidence distributions and confidence intervals}

As (approximate) confidence distributions might be obtained from likelihoods by probability transforming the deviance function $D$, (approximate) likelihoods may be obtained from confidence distributions by the opposite quantile transformation of the confidence curve. More specifically, if the cumulative distribution of the (profile) deviance function evaluated at the value of the parameter $\theta$ which governs the distribution of the data is $F_{\theta}, c c(\theta, X)=F_{\theta}(D(\theta, X))$ is the obtained confidence curve. By Wilks Theorem, the $\chi^{2}$ distribution approximates $F_{\theta}$ well. With $\operatorname{dim}(\theta)=p$ and with $K_{p}$ being the cumulative $\chi^{2}$ distribution,

$$
L_{c}(\theta, X)=\exp \left[-\frac{1}{2} K_{p}^{-1}(c c(\theta, X))\right]
$$

is what Schweder and Hjort (2015) call the confidence likelihood. Efron (1993) introduced his implied likelihood exp $\left[-\frac{1}{2} \Phi^{-1}(C(\theta, X))^{2}\right]$, which agrees with the confidence likelihood in case $p=1$ and $c c(\theta)=|1-2 C(\theta)|$.

Integrating the information in independent confidence distributions might be achieved by adding their confidence log-likelihoods. When the independent confidence distributions are for the same one-dimensional parameter, they might also be integrated by adding their normal scores (Xie and Singh 2013). Since $\Phi^{-1}\left(C_{i}\left(\theta, X_{i}\right)\right) \sim N(0,1)$ when $\theta=\theta_{0}$, the true value, $\Phi\left(n^{-1 / 2} \sum_{i=1}^{n} \Phi^{-1}\left(C_{i}\left(\theta, X_{i}\right)\right)\right.$ is a confidence distribution for $\theta$ based on the combined evidence.

When only independent confidence intervals are available, one might try to combine their evidence by first estimating a confidence curve behind each confidence interval. 
Let the degree of confidence be $\alpha=1-\epsilon$, and assume that in addition to the interval $(\theta(\epsilon / 2), \theta(1-\epsilon / 2))$ a point estimate is available, which is assumed to be the confidence median $\hat{\theta}=\theta(1 / 2)$. We thus have three confidence quantiles available. In the symmetric case when $\theta(1-\epsilon / 2)-\theta(1 / 2)=\theta(1 / 2)-\theta(\epsilon / 2)$ we might assume that the confidence interval is obtained from a normal pivot with standard error $s$. Then $s=(\theta(1-\epsilon / 2)-$ $\theta(1 / 2)) / \Phi^{-1}(1-\epsilon / 2)$ and $L_{c}(\theta)=\exp \left[-\frac{1}{2}\left(\frac{\theta-\hat{\theta}}{s}\right)^{2}\right]$. In the asymmetric case with a positive parameter we might assume that the underlying confidence distribution is a transformed normal, $C(\theta)=\Phi\left(\frac{h(\theta)-h(\hat{\theta})}{s}\right)$ with $h(\theta)=\operatorname{sign}(a) \theta^{a}$. Then $s$ and the exponent $a$ are found by solving the two equations $\theta(1-\epsilon / 2)^{a}-\hat{\theta}^{a}=s \Phi^{-1}(1-\epsilon / 2)$ and $\hat{\theta}^{a}-\theta(\epsilon / 2)^{a}=s \Phi^{-1}(1-$ $\epsilon / 2)$. Under these assumptions the confidence likelihood is $L_{c}(\theta)=\exp \left[-\frac{1}{2}\left(\frac{\theta^{a}-\hat{\theta}^{a}}{s}\right)^{2}\right]$. If $a$ comes out close to zero the log transformation might apply. This approach is indeed quick and dirty. Better approximations might be obtained if more information is available regarding the confidence interval.

Example 7 (Effective population size for cod) The effective population size $N_{e}$ of a given population is the size of a hypothetical stable population that maintains the same genetic variability as the actual population over the generations. In a study of a cod population a point estimate and a 95\% confidence interval for $N_{e}$ was found from genetic data by a relatively complex method involving jackknifing, see Knutsen et al. (2011). A $95 \%$ confidence interval was also found for the actual population size $N$, resulting from the standard linear normal pivot. The results for $N_{e}$ and $N$ are stochastically independent. The confidence quantiles are as follows.

\begin{tabular}{c|ccc} 
& $2.5 \%$ & $50 \%$ & $97.5 \%$ \\
\hline$N_{e}$ & 106 & 198 & 1423 \\
$N$ & 800 & 1847 & 2893 \\
\hline
\end{tabular}

From these data a confidence interval is sought for the ratio $\psi=N_{e} / N$. This ratio can be used to estimate the variance in number of reproductive off-springs, which is hard to estimate by other methods. The confidence log likelihood for $N$ is simply $\ell_{c}(N)=$ $-\frac{1}{2}\left(\frac{N-1847}{534}\right)^{2}$ since we find $s=534$. For $N_{e}$ we chose the linear normal model in power transformed parameter. Solving the two equations we find $a=-0.992$ and $s=.00233$. Using $a=-1$ the confidence likelihood is $l_{c}\left(N_{e}\right)=-\frac{1}{2}\left(\frac{N_{e}^{-1}-198^{-1}}{0.00233}\right)^{2}$.

The confidence curve for $\psi$ is found by probability-transforming the confidence deviance which is found by profiling the joint confidence log likelihood for $\left(N, N_{e}\right)$. It is shown in Figure 3. Since by definition $N_{e} \leq N$ the 95\% confidence interval is $(0.0467,1)$. The confidence distribution has an atom of some 0.025 at $\psi=1$. The confidence curve has a long tail to the right. A confidence interval of high level of confidence will thus be quite sensitive to the level with respect to its right endpoint.

\section{$5 \quad$ Bayes and confidence, best friends forever?}

Berger (2006) argues that objective Bayesian methods are the most promising route to the unification of Bayesian and frequentist methodologies. Meng (2014, IMS Bulletin) 


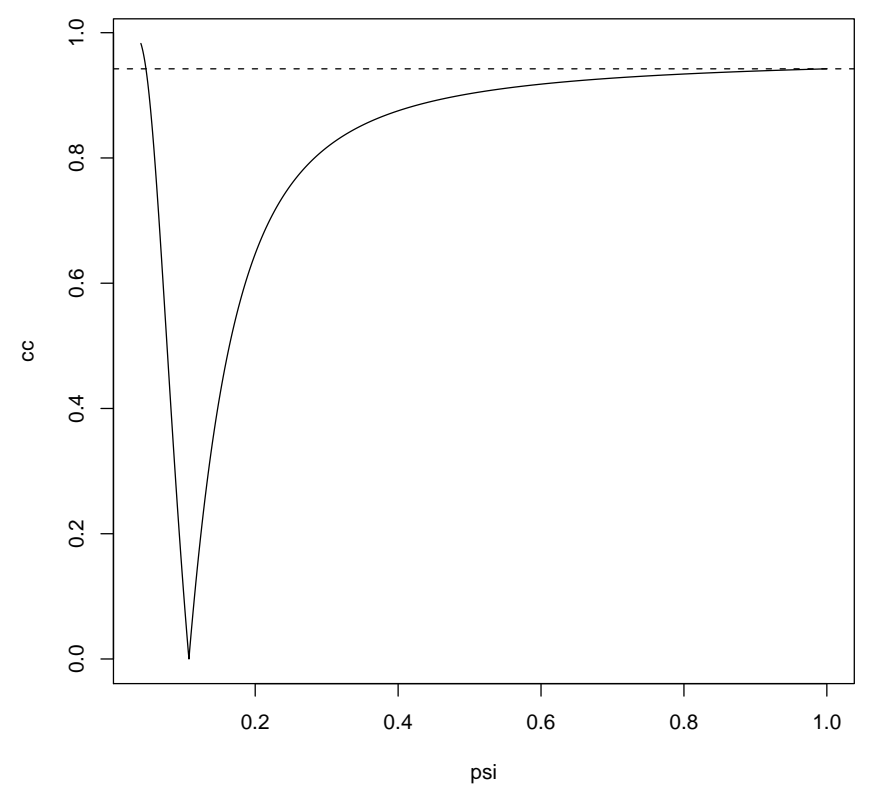

Figure 3: Confidence curve for $\psi=N_{e} / N$. The $95 \%$ level is shown as a dashed line.

follows suit and asks "Can our desire to find a unification of Bayesian, Frequentist and Fiducial (BFF) perspectives do the same trick, allowing all of us to thrive under one roof as BFFs (Best Friends Forever)?" Fiducial distributions are confidence distributions in one dimension, and provides approximate confidence distributions in higher dimensions. We might thus ask whether objective Bayes and confidence might be "best friends forever". The two methodologies have indeed much in common.

First, Confidence distributions are invariant to monotonic transformations of the parameter. Only Bayesian posteriors based on invariant priors might thus agree with a confidence distribution. Bayesian posteriors based on Jeffreys priors are invariant. Objective Bayes methods, based on Jeffreys priors or on reference priors (Berger and Bernardo 1992), are often exact or approximate confidence distributions. Lindley (1958) proved that for a scalar parameter the Bayesian posterior is exactly a confidence distribution if and only if the model might be transformed to a location model, and the prior is of Jeffreys type.

Second, both confidence distributions and objective Bayes methods produce epistemic distributions without any other input than the observed data and the model. Confidence distributions have been named posteriors without priors (Schweder and Hjort 2003).

Third, although confidence distribution might be obtained in non-parametric and semi-parametric models, see e.g. Schweder and Hjort (2016, Chapter 11), it is mainly in parametric models the method has been applied. Objective Bayes is also primarily concerned with parametric models.

Fourth, in the usual case with a parameter of special interest, a focus parameter, 
both methods aims at a good epistemic distribution for that parameter. The confidence distribution will often be based on the profile likelihood or the conditional likelihood. The reference prior actually aims for the focus parameter. Bernardo's reference prior is constructed to minimize the asymptotic missing information (Berger and Bernardo 1992). Any prior adds a piece of information to the posterior distribution, a piece that does not come from the data or the model. But in cases when there are no external reasons to chose a specific prior, other than the focus, this piece of information is missing. The reference prior is the one that minimizes this information, in a specific sense, and asymptotically as the data gets more and more informative while the parameter is kept constant.

Fifth, and not least, objective Bayes methods have good coverage properties. With $\theta_{b}(\epsilon, x)$ being the $\epsilon=1-\alpha$ quantile in the Bayesian posterior distribution based on data $x$ for the one dimensional parameter $\theta,\left(\theta_{b}(\epsilon / 2, X), \theta_{b}(1-\epsilon / 2, X)\right)$ is a second order correct confidence interval at level $\alpha$ when the prior is of the Jeffreys type (Sweeting 2001). Sweeting actually calls a Baysian method for objective if it is well calibrated, i.e. has good coverage properties in the frequentist sense. His objective Bayesian posteriors are thus approximate confidence distributions.

\section{Concluding remarks}

A particular measurement is to be trusted when made by a reliable instrument. Confidence distribution is a statistical instrument. Since confidence distributions in repeated use are uniformly distributed at the true values of the respective parameters, and thus that any interval between two confidence quantiles covers the true value with the corresponding confidence, the confidence distributions are the "simple and interpretable summaries of what can reasonably be learned from the data (and an assumed model)" (Cox 2013). I agree with Hampel (2006) that these distributions should be regarded as epistemic probability distributions also by frequentists. Our statistical thinking, and also our communication with students and substantive scientists would be eased by acknowledging both aleatory and epistemic probability. I actually think that even the orthodox frequentist, after having worked hard at an applied project ending up with a confidence interval for the parameter of interest, say the Newtonian gravitational constant, in his heart thinks of the level of confidence as the probability that the parameter really is within the bounds of the obtained confidence interval.

\section{References}

[1] Arnauld, A. and Nicole, P. (1662) La logique ou l'art de penser (Logic Or the Art of Thinking, edited by Buroker, J.V., Cambridge University Press, 1996)

[2] Berger, J.O. and Bernardo, J.M. (1992) On the development of reference priors. Bayesian statistics. 4: $35-60$.

[3] Berger, J.O. (2006) The case for objective Bayesian analysis. Bayesian analysis, 1: 385-402. 
[4] Birnbaum, A. (1961). Confidence curves: An omnibus technique for estimation and testing statistical hypotheses. Journal of the American Statistical Association, 56:246249.

[5] Boole, G. (1854). The Laws of Thought [reprinted by Dover, New York, 1958]. Macmillan, London.

[6] Brazzale, A. R., Davison, A. C., and Reid, N. (2007). Applied Asymptotics: Case Studies in Small-Sample Statistics. Cambridge University Press, Cambridge.

[7] Breiman, L. (1992). The little bootstrap and other methods for dimensionality reduction in regression: X-fixed prediction error. Journal of the American Statistical Association, 87:738-754.

[8] Cisewski,J. and Hannig, J. (2012) Generalized fiducial inference for normal linear mixed models. The Annals of Statistics, 40: 2102-2127.

[9] Cox, D. R. (1958). Some problems with statistical inference. The Annals of Mathematical Statistics, 29:357-372.

[10] Cox, D. R. (2013). Discussion of M. Xie and K. Singh's paper. International Statistical Review, 81:40-41.

[11] Efron, B. (1993). Bayes and likelihood calculations from confidence intervals. Biometrika, 80:3-26.

[12] Efron, B. (1998). R.A. Fisher in the 21st century [with discussion and a rejoinder]. Statistical Science, 13:95-122.

[13] Fisher, R. A. (1930). Inverse probability. Proceedings of the Cambridge Philosophical Society, 26:528-535.

[14] Fisher, R. A. (1973). Statistical Methods and Scientific Inference (3rd ed.). Hafner Press. Extended version of the 1956 edition.

[15] Fraser, D. A. S. (2011). Is Bayes posterior just quick and dirty confidence? [with discussion and a rejoinder]. Statistical Science, 26:249-316.

[16] Guttman, L. (1985). The illogic of statistical inference for cumulative science. Applied Stochastic Models and Data Analysis, 1:3-9.

[17] Hacking, I. M. (1965). Logic of Statistical Inference. Cambridge University Press, Cambridge.

[18] Hacking, I.M. (1975). The Emergence of Probability: A Philosophical Study of Early Ideas About Probability, Induction and Statistical Inference. Cambridge University Press, Cambridge.

[19] Hald, A. (1990). A History of Probability and Statistics and Their Applications Before 1750. John Wiley \& Sons, New York.

[20] Hampel, F. (2006). The proper fiducial argument. In Ahlswede, R., editor, General Theory of Information Transfer and Combinatorics, Lecture Notes in Computer Science, No. 4123, pages 512-526. Springer-Verlag, Heidelberg. 
[21] Hannig, J., Iyer, H., Lai, R.C.S. and Lee T.C.M. (2016). Generalized Fiducial Inference: A Review and New Results. Journal of the American Statistical Association, 111:1346-1361.

[22] IPCC (2013). Climate Change 2013: Fifth Assessment Report of the Intergovernmental Panel on Climate Change. United Nations, New York.

[23] Kalbfleisch, J. G. and Sprott, D. A. (1967) Fiducial probability. Statistische Hefte, (1967-06-01) 8: 99-109.

[24] Knutsen, H., Olsen, E. M., Jorde, P. E., Espeland, S. H., Andre, C. and Stenseth, N. C. (2011). Are low but statistically significant levels of genetic differentiation in marine fishes 'biologically meaningful'? A case study of coastal Atlantic cod. Molecular Ecology, 20:768-783.

[25] Lindley, D. V. (1958). Fiducial distributions and Bayes' theorem. Journal of the Royal Statistical Society, Series B, 20:102-107.

[26] Martin, R. and Liu C. (2014) A note on p-values interpreted as plausibilities. Statistica Sinica, 24:1703-1716

[27] Martin, R. and Liu C. (2015). Inferential models: Reasoning with Uncertainty Monographs in Statistics and Probability Series. Chapman \& Hall/CRC Press.

[28] Milyukov, V. and Fan, S.-H. (2012). The Newtonian gravitational constant: Modern status of measurement and the new CODATA value. Gravitation and Cosmology, $18: 216-224$.

[29] Neyman, J. (1934). On the two different aspects of the representative method: The method of stratified sampling and the method of purposive selection. Journal of the Royal Statistical Society, Series A, 97:558-625.

[30] Pitman, E. J. G. (1939). The estimation of location and scale parameters of a continuous population of any given form. Biometrika, 30:391-421.

[31] Pitman, E. J. G. (1957). Statistics and science. Journal of the American Statistical Association, 52: 322-330.

[32] Reid, N. and Cox, D. R. (2015). On some principles of statistical inference. International Statistical Review, 83: 293-308.

[33] Schweder, T. and Hjort, N. L. (2003). Frequentist analogues of priors and posteriors. In Stigum, B. (ed.),Econometrics and the Philosophy of Economics: Theory Data Confrontation in Economics, pp. 285-217. Princeton University Press, Princeton, NJ.

[34] Schweder, T. and Hjort, N. L. (2016). Confidence, Likelihood, Probability: Statistical inference with confidence distributions. Cambridge University Press. In press.

[35] Sweeting T.J. (2001) Coverage probability bias, objective Bayes and the likelihood principle. Biometrika, 88: 657-675.

[36] Trafimow, D., and Marks, M. (2016). Editorial. Basic and Applied Social Psychology, $38,1-2$. 
[37] Wasserstein R. L. and Lazar N. A (2016). The ASA's statement on pvalues: context, process, and purpose. The American StatisticianStatistician, DOI: 10.1080/00031305.2016.1154108

[38] Xie, M. and Singh, K. (2013). Confidence distribution, the frequentist distribution estimator of a parameter: a review [with discussion and a rejoinder]. International Statistical Review, 81:3-39. 